\title{
Serum Albumin Levels and Clinical Outcomes Among Ischemic Stroke Patients Treated with Endovascular Thrombectomy
}

This article was published in the following Dove Press journal: Neuropsychiatric Disease and Treatment

Jie Gao, ',* Ying Zhao, ',* Mingyang Du, ${ }^{2, *}$ Hongquan Guo, ${ }^{3}$ Ting Wan, ' Min $\mathrm{Wu}, \mathbb{D}^{3}$ Ling Liu, 'Huaiming Wang, (D) ${ }^{1,4}$ Qin Yin,' Xinfeng Liu'

'Department of Neurology, Jinling Hospital, Medical School of Nanjing University, Nanjing, Jiangsu, 210000, People's Republic of China;

${ }^{2}$ Cerebrovascular Disease Treatment Center, Nanjing Brain Hospital Affiliated to Nanjing Medical University, Nanjing, Jiangsu, 210000, People's Republic of

China; ${ }^{3}$ Department of Neurology, Jinling Hospital, Southern Medical University, Nanjing, 210000, People's Republic of China; ${ }^{4}$ Department of Neurology, The 80th Group Army Hospital, Weifang, Shandong, 26I000, People's Republic of China

*These authors contributed equally to this work

Correspondence: Huaiming Wang Department of Neurology, Jinling Hospital, Medical School of Nanjing University, No. 305 East Zhongshan Road, Nanjing, 210002, Jiangsu Province,

People's Republic of China

$\mathrm{Tel}+862584801861$

Fax +862584805I69

Email wanghm89@163.com

Qin Yin

Department of Neurology, Jinling Hospital, Medical School of Nanjing University, No. 305 East Zhongshan Road, Nanjing, 210002, Jiangsu Province,

People's Republic of China

$\mathrm{Tel}+86258480$ I86I

Fax +862584805169

Email yinqin9912@163.com
Background and Aims: Albumin levels have been reported to be associated with all-cause and cardiovascular mortality. The aim of this study was to investigate the association between serum albumin and prognosis of ischemic stroke patients after endovascular thrombectomy (EVT) treatment.

Methods: Patients with EVT due to large artery occlusion in anterior circulation were selected from ACTUAL (endovascular treatment for acute anterior circulation) ischemic stroke multicenter registry in China. Serum albumin levels were measured within $24 \mathrm{~h}$ of admission. The primary outcome was poor functional outcome (modified Rankin scale score of 3-6) at three months. Secondary outcomes were symptomatic intracranial hemorrhage (sICH) and three-month mortality.

Results: A total of 605 patients (mean age, 64.2 years; $59.3 \%$ male) were enrolled. Up to three months after stroke, 342 patients (56.5\%) developed poor functional outcome. After multivariate adjustment for demographic characteristics, National Institutes of Health stroke score, and other potential confounders, the odds ratio for the lowest tertile of serum albumin levels was $2.43(95 \% \mathrm{CI}, 1.18-5.01 ; P=0.046)$ for poor functional outcome, compared with the highest category. Restricted cubic spline regression demonstrated a linear association between albumin levels and poor functional outcome $(P$ for linearity=0.017). Subgroup analyses further confirmed these results. Similar significant findings were also found in the association of serum albumin with mortality, but not with sICH.

Conclusion: Decreased serum albumin levels were independently associated with poor prognosis at 90 days after acute large vessel occlusion stroke in anterior circulation treated with EVT.

Keywords: albumin, ischemic stroke, endovascular treatment, prognosis

\section{Introduction}

The trajectory of an ischemic stroke patient due to large vessel occlusion is fundamentally altered by endovascular thrombectomy (EVT). ${ }^{1-5}$ Based on the current guideline for management of ischemic stroke, ${ }^{6}$ EVT is the standard care for patients with artery occlusion in anterior circulation. However, only half of the patients receiving EVT had functional independence (modified Rankin Scale, mRS, $0-2$ ) at 90 days, and 10\% were neurologically normal according to a recent metaanalysis of EVT trials enrolling 1287 participants. ${ }^{7}$ Therefore, identifying influencing factors for clinical outcomes is of vital importance for continuously improving the prognosis of patients after EVT. 
Albumin is synthesized in the liver and regulates the colloidal osmotic pressure. It can also inhibit platelet aggregation, ${ }^{8}$ mediate systemic inflammation, ${ }^{9}$ and exert antioxidant activity. ${ }^{10}$ Cumulative evidence has shown the protective effects of serum albumin in many diseases such as hypertension, ${ }^{11}$ carotid atherosclerosis, ${ }^{12}$ and cardiovascular diseases. ${ }^{13}$ In addition, a reduction in albumin level is negatively associated with ischemic stroke severity. ${ }^{14,15}$ Data from an experimental study further confirmed the neuroprotection of albumin and demonstrated that human albumin therapy significantly improves neurologic function, and reduces infarction volume and brain swelling in animal models with acute focal ischemic stroke. ${ }^{16}$ The role of albumin as a neuroprotectant has been assessed in humans in the ALIAS (albumin in acute stroke) trial. However, the Phase III clinical trial did not confirm that high-dose albumin treatment can improve neurologic function in acute ischemic stroke patients. ${ }^{17}$ More studies are needed to investigate the effects of serum albumin in the prognosis of ischemic stroke, especially in patients with different etiology and therapeutic types. Hypotension before reperfusion may compromise collateral flow and lead to a poor outcome in larger vessel occlusive stroke. To date, data are scarce regarding the prognostic value of serum albumin in ischemic stroke patients after EVT. This study aimed to explore the relationship between serum albumin levels and prognosis at 90 days after EVT in a large cohort of Chinese patients.

\section{Methods}

\section{Study Design and Participants}

Eligible patients with EVT were selected from the ACTUAL (endovascular treatment for acute anterior circulation) ischemic stroke registry between January 2014 and June 2016. The ACTUAL is a registry of EVT for anterior circulation large vessel occlusion stroke in 21 hospitals across China. ${ }^{18}$ Patients were recruited in this study if they met all of the following: (1) aged 18 years or older; (2) had pre-stroke mRS score $\leq 2$; (3) had occlusion at internal carotid artery or middle cerebral artery confirmed by computed tomographic angiography, magnetic resonance angiography, or digital subtracted angiography.

We excluded patients treated with intra-arterial thrombolysis alone, treated with devices other than a stent-like retriever, had pre-stroke mRS score $>2$, or diagnosed with malignant tumor, autoimmune disease, severe renal insufficiency, hepatic disease, and heart failure. All procedures performed in studies involving human participants were in accordance with the ethical standards of the 1964 Helsinki Declaration and its later amendments, and approved by the Jingling Hospital Ethics Committee. Due to its retrospective nature; patient consent was waived. Patients data confidentiality was maintained in Jingling Hospital and each participating center.

\section{Data Collection}

We collected demographic characteristics, cardiovascular risk factors (hypertension, diabetes, hyperlipidemia, coronary heart disease, and atrial fibrillation), clinical data (blood pressure, prior intravenous thrombolysis, stroke severity, pre-stroke mRS, and stroke etiology), imaging data (occlusion site, collateral flow, the Alberta Stroke Program Early CT Score, ASPECTS), and proceduralrelated characteristics (operation modes, total passes of stent retriever, recanalization, onset to treatment time, OTT, and puncture to recanalization time). Stroke severity after admission was evaluated by using National Institutes of Health Stroke Scale (NIHSS). ${ }^{19}$ Stroke subtype was classified according to the criteria of Trial of Org 10,172 in acute stroke treatment. ${ }^{20}$ Collateral circulation status was evaluated using the American Society of Interventional and Therapeutic Neuroradiology/ Society of Interventional Radiology (ASITN/SIR) grading system. ${ }^{21}$ We defined the ASITN/SIR $0-1$ as poor collateral circulation. Vascular recanalization was defined as modified thrombolysis in cerebral infarction score of $2 \mathrm{~b}$ or $3 .{ }^{22}$ The symptomatic intracranial hemorrhage (sICH) was detected according to the criteria of the Heidelberg Bleeding Classification within $72 \mathrm{~h}$ after EVT. $^{23}$

Follow-up of mRS at three months after stroke onset was accomplished by each center, either by telephone or by outpatient visit. The primary study outcome was poor functional outcome, which defined as mRS score of 3-6. sICH and three-month mortality were the secondary outcomes of this study.

\section{Albumin Concentrations Measurement}

Peripheral blood samples were taken from the antecubital vein from each subject within $24 \mathrm{~h}$ of admission. Albumin concentrations were measured using automated immunoturbidimetric assay. Laboratory data included blood glucose, total cholesterol, triglyceride, high-density lipoprotein, low-density lipoprotein, and hypersensitive C-reactive protein (Hs-CRP) were also recorded. 


\section{Statistical Analysis}

Continuous variables were presented as mean (SD) and median (interquartile range), and categorical variables as number (percentage). Participants were divided into three groups according to the serum albumin levels tertile. Differences in baseline characteristics between groups were analyzed using independent sample $t$-tests, MannWhitney $U$-tests, one-way analysis of variance, and Kruskal-Wallis $H$-test for continuous variables, and the chi-squared test or Fisher's exact test for categorical variables, as appropriate.

An association was estimated by calculating OR and $95 \% \mathrm{CI}$. The effect of serum albumin on mRS score was analyzed using ordinal logistic regression model. Multivariable logistic regression analyses with two models were conducted to investigated the risk factors of clinical outcomes. Model 1 was adjusted for age and sex, and model 2 further adjusted for variables with $P<0.1$ in univariate analysis of clinical outcomes. In addition, we further used restricted cubic splines to evaluate the pattern and magnitude of association between serum albumin and clinical outcomes, with four knots defined at the 5th, 35th, 65th, and 95th percentiles of albumin levels. ${ }^{24}$

We also performed subgroup analyses to assess the robustness of association between serum albumin levels and primary outcome. Interactions between serum albumin and subgroup variables on the primary outcome were tested in the models with interaction terms by the likelihood ratio test, adjusting for the covariates in model 2 unless the variable was used as a subgroup variable. Statistical analysis was performed using SPSS software, version 22.0 (IBM Corporation, Armonk, NY, USA) and $\mathrm{R}$ statistical software version 3.6.2, and 2-tailed $P$-value $<0.05$ were considered statistically significant.

\section{Results}

In a total of 698 patients, we excluded 66 patients treated with intra-arterial thrombolysis alone or devices other than a stent-like retriever, three patients with anterior cerebral artery occlusion, seven patients with pre-mRS score $>2,17$ patients with preexisting renal insufficiency, hepatic insufficiency, heart failure, autoimmune disease, and cancer. Finally, 605 patients (mean age, 64.2 years; $59.3 \%$ male) were included in the study. Patients were divided into three groups according to the albumin tertile: first tertile $(<37.3$ $\mathrm{g} / \mathrm{L})$, second tertile $(37.3 \mathrm{~g} / \mathrm{L}-40.2 \mathrm{~g} / \mathrm{L})$, and third tertile $(>40.2 \mathrm{~g} / \mathrm{L})$. The demographics and clinical characteristics stratified by the albumin tertile were presented in Table 1 . The participants with lower serum albumin levels tended to be older and had higher systolic blood pressure, baseline NIHSS score, Hs-CRP, prevalence of atrial fibrillation, and poorer collateral status than those with higher serum albumin. There was a significant difference regarding the overall distribution of mRS scores across the albumin levels tertile in ordinal regression analysis (lowest tertile vs highest tertile: adjusted OR, $1.62 ; 95 \% \mathrm{CI}, 1.02-2.43, P$ for trend $=0.039$; Figure 1).

After three months of follow-up, 342 participants (56.5\%) experienced poor functional outcome. Compared with participants without primary outcome, those who developed primary outcome were more likely to be older, female, and had higher systolic blood pressure, blood glucose, Hs-CRP, baseline NIHSS score, passes of stent retrieve, and prevalence of atrial fibrillation, cardioembolic stroke, poorer collateral status, internal carotid artery occlusion, sICH, and recanalization. Patients with poor outcome at three months had lower baseline ASPECTS score (Table 2). The results of comparison of baseline data according to patients with and without secondary outcomes were showed in Table S1 and Table S2.

The cumulative rates of poor functional outcome at 90 days according to the albumin tertile (from lowest to highest) were $69.5 \%, 54.9 \%$, and $45.0 \%$, respectively (Table $3)$. In univariate logistic analysis, the lowest tertile of serum albumin (compared with the highest tertile) was associated with increased risk of poor functional outcome (OR, 2.78, 95\% CI 1.85-4.18; $P$ for trend $=0.001)$ and mortality $(\mathrm{OR}, \quad 2.97, \quad 95 \% \mathrm{CI}, \quad 1.82-4.85 ; \quad P$ for trend $=0.001$ ). Similar significant findings were observed when the albumin levels were added as a continuous variable. These associations remained significant after adjustment for demographic characteristic, baseline NIHSS score, baseline ASPECTS score, and other potential confounders. In addition, we assessed the potential correlation between the levels of albumin and clinical outcomes using restricted cubic splines with four knots. The result further indicated a linear dose-response association between serum albumin and clinical outcomes (poor functional outcome, $P$ for linearity $=0.017$, Figure $2 \mathrm{~A}$; death, $P$ for linearity $=0.014$, Figure $2 \mathrm{~B}$ ). No such relationship was found for albumin levels with sICH (all $P>0.05$, Figure 2C).

In subgroup analyses stratified by age, sex, hypertension, diabetes, atrial fibrillation, prior intravenous thrombolysis, baseline NIHSS score, baseline ASPECTS score, OTT time, recanalization and cause of stroke, the modest 
Table I Demographics and Clinical Characteristics Stratified by the Tertile of Albumin Levels

\begin{tabular}{|c|c|c|c|c|c|}
\hline Variables & All Patients & $\begin{array}{l}\text { Ist Tertile, } \\
<37.3 \mathrm{~g} / \mathrm{L}\end{array}$ & $\begin{array}{l}\text { 2nd Tertile, } 37.3 \mathrm{~g} / \mathrm{L} \\
-40.2 \mathrm{~g} / \mathrm{L}\end{array}$ & $\begin{array}{l}\text { 3rd Tertile, } \\
>40.2 \mathrm{~g} / \mathrm{L}\end{array}$ & $\begin{array}{l}P \text { for } \\
\text { trend }\end{array}$ \\
\hline No. of patients & 605 & 203 & 202 & 200 & \\
\hline \multicolumn{6}{|l|}{ Demographic characteristics } \\
\hline Age, years & $64.2 \pm 12.4$ & $66.9 \pm 11.8$ & $64.3 \pm 13.1$ & $61.4 \pm 11.6$ & 0.001 \\
\hline Male, n (\%) & $359(59.3)$ & $115(56.7)$ & $124(61.4)$ & $120(60.1)$ & 0.608 \\
\hline \multicolumn{6}{|l|}{ Vascular risk factors, n (\%) } \\
\hline Hypertension & $374(61.8)$ & $|3|(64.5)$ & $115(56.9)$ & $128(64.0)$ & 0.214 \\
\hline Diabetes mellitus & $104(17.2)$ & $40(19.7)$ & $34(16.8)$ & $30(15.0)$ & 0.451 \\
\hline Hyperlipidemia & $54(8.9)$ & $16(7.9)$ & $18(8.9)$ & $20(10.0)$ & 0.757 \\
\hline Atrial fibrillation & 241 (398.) & $96(47.3)$ & $75(37.1)$ & $70(35.2)$ & 0.028 \\
\hline Coronary heart disease & $130(21.5)$ & $48(23.6)$ & $43(21.4)$ & $39(19.5)$ & 0.598 \\
\hline \multicolumn{6}{|l|}{ Clinical data } \\
\hline Systolic blood pressure, $\mathrm{mmHg}$ & $146.6 \pm 25.8$ & $150.6 \pm 28.5$ & $145.3 \pm 26.3$ & $143.8 \pm 22.1$ & 0.020 \\
\hline Diastolic blood pressure, $\mathrm{mmHg}$ & $84.2 \pm 14.1$ & $84.3 \pm 14.3$ & $84.9 \pm 14.7$ & $83.6 \pm 13.3$ & 0.656 \\
\hline Time from onset to treatment, $\min$ & $268.0(200.0,345.0)$ & $270.0(205.0,350.0)$ & $250.0(190.0,345.0)$ & $270.0(207.0,340.0)$ & 0.489 \\
\hline $\begin{array}{l}\text { Time from puncture to } \\
\text { recanalization, } \min \end{array}$ & $75.0(54.0,103.0)$ & $77.0(56.0,113.0)$ & $75.0(52.0,102.0)$ & $75.0(54.0,99.0)$ & 0.208 \\
\hline Baseline NIHSS, score & $16.0(12.0,21.0)$ & $18.0(13.0,23.0)$ & $16.0(12.0,20.0)$ & $15.0(12.0,19.0)$ & 0.001 \\
\hline Baseline ASPECTS, score & $9.0(8.0,10.0)$ & $9.0(8.0,10.0)$ & $9.0(8.0,10.0)$ & $9.0(8.0,10.0)$ & 0.557 \\
\hline Cause of stroke, n (\%) & & & & & 0.100 \\
\hline Atherosclerotic & $27 \mid(44.9)$ & 79 (38.9) & $98(48.5)$ & $94(47.0)$ & \\
\hline Cardioembolic & $289(46.2)$ & $112(55.2)$ & $90(44.6)$ & $87(43.5)$ & \\
\hline Others & $45(7.4)$ & $12(5.9)$ & $14(6.9)$ & $19(9.5)$ & \\
\hline Poor collateral status & $283(46.8)$ & $107(53.0)$ & $100(49.5)$ & $76(38.2)$ & 0.007 \\
\hline Procedural modes, n (\%) & & & & & 0.417 \\
\hline Stent retriever only & $291(48.1)$ & $90(44.3)$ & $100(49.5)$ & $101(50.5)$ & \\
\hline Stent retriever with rescue therapy ${ }^{a}$ & $314(51.9)$ & $113(55.7)$ & $102(50.5)$ & $99(49.5)$ & \\
\hline Passes of stent retriever & $1.0(1.0,2.0)$ & $2.0(1.0,3.0)$ & $1.0(1.0,2.0)$ & $1.0(1.0,2.0)$ & 0.107 \\
\hline Vascular occlusion site, $\mathrm{n}(\%)$ & & & & & 0.925 \\
\hline Internal carotid artery & $249(4 I .2)$ & $84(41.4)$ & $81(40.1)$ & $84(42.0)$ & \\
\hline Middle cerebral artery & $356(58.8)$ & $119(58.6)$ & $|2|(59.5)$ & $116(58.0)$ & \\
\hline Prior IVT, n (\%) & $199(32.9)$ & $67(33.0)$ & $66(32.7)$ & $66(33.0)$ & 0.997 \\
\hline sICH, n (\%) & $93(15.4)$ & $35(17.2)$ & $32(15.8)$ & $26(13.0)$ & 0.486 \\
\hline Recanalization, n (\%) & $53(82.6)$ & 161 (79.3) & $170(84.2)$ & $169(84.5)$ & 0.305 \\
\hline
\end{tabular}

(Continued) 
Table I (Continued).

\begin{tabular}{|c|c|c|c|c|c|}
\hline Variables & All Patients & $\begin{array}{l}\text { Ist Tertile, } \\
<37.3 \mathrm{~g} / \mathrm{L}\end{array}$ & $\begin{array}{l}\text { 2nd Tertile, } 37.3 \mathrm{~g} / \mathrm{L} \\
-40.2 \mathrm{~g} / \mathrm{L}\end{array}$ & $\begin{array}{l}\text { 3rd Tertile, } \\
>40.2 \mathrm{~g} / \mathrm{L}\end{array}$ & $\begin{array}{l}P \text { for } \\
\text { trend }\end{array}$ \\
\hline \multicolumn{6}{|l|}{ Laboratory data } \\
\hline Total cholesterol, $\mathrm{mmol} / \mathrm{L}$ & $4.3 \pm 1.1$ & $4.2 \pm 1.2$ & $4.3 \pm 1.2$ & $4.4 \pm 0.9$ & 0.107 \\
\hline Triglyceride, $\mathrm{mmol} / \mathrm{L}$ & $1.0(0.7,1.5)$ & $1.0(0.8,1.5)$ & $1.0(0.7,1.5)$ & I.I $(0.8,1.5)$ & 0.658 \\
\hline Low-density lipoprotein, mmol/L & $2.5(1.9,3.1)$ & $2.3(1.9,3.0)$ & $2.5(1.9,3.2)$ & $2.5(2.1,3.1)$ & 0.115 \\
\hline High-density lipoprotein, mmol/L & $1.2 \pm 0.4$ & $1.2 \pm 0.5$ & $1.2 \pm 0.5$ & $1.2 \pm 0.3$ & 0.995 \\
\hline Blood glucose, $\mathrm{mmol} / \mathrm{L}$ & $7.7 \pm 3.4$ & $8.1 \pm 3.8$ & $7.7 \pm 3.6$ & $7.3 \pm 2.7$ & 0.065 \\
\hline Hs-CRP, mg/L & $7.6(2.5,24.5)$ & $9.1(2.9,29.0)$ & $8.6(2.8,26.5)$ & $5.4(1.9,13.3)$ & 0.034 \\
\hline
\end{tabular}

Note: aRescue therapy includes balloon angioplasty, permanent implantation of stent, intra-arterial thrombolysis or intra-arterial tirofiban infusion.

Abbreviations: ASPECTS, the Alberta Stroke Program Early Computed Tomography Score; Hs-CRP, hypersensitive C-reactive protein; IVT, intravenous thrombolysis; NIHSS, National Institutes of Health Stroke Scale; sICH, symptomatic intracranial hemorrhage.

negative associations between serum albumin and risk of poor functional outcome were observed in almost all subgroups and reached statistically significance in several subgroups. Moreover, no significant interaction was detected between serum albumin levels and these interesting factors on the poor functional outcome (all $P$-value for interaction $>0.05$, Figure 3).

\section{Discussion}

Our study investigated the association between serum albumin levels at baseline and prognosis of ischemic stroke treated with EVT. We found that lower serum albumin levels were independently associated with increased risk of the poor functional outcome at 90 days, and there was a negative linear dose-response relationship between them. Subgroup analyses further confirmed these associations. No correlation was found between albumin levels and sICH.
Hypoalbuminemia was a concomitant syndrome in strokes. ${ }^{25-27}$ Davalos et al $^{25}$ measured albumin levels within $24 \mathrm{~h}$ after symptoms onset in 104 acute strokes patients, and showed that hypoalbuminemia $(<35 \mathrm{~g} / \mathrm{L})$ was observed in $7.7 \%$ of patients. Another cohort study of 705 ischemic and hemorrhagic stroke found that hypoalbuminemia was occurred in $45.5 \%$ of patients. ${ }^{26}$ In our present study, $122(20.2 \%)$ patients obtained a serum albumin levels $<35 \mathrm{~g} / \mathrm{L}$. These discrepancies in the hypoalbuminemia rates between our study and previous reports might be explained, at least in part, by differences concerning the study populations and study design, especially time of blood sampling. Hypoalbuminemia in stroke patients could be a result of malnutrition and/or underlying disease processes such as renal or hepatic insufficiency, and malignancy. To keep the homogeneity of the enrolled patients, we excluded patients diagnosed with malignant tumor, severe renal

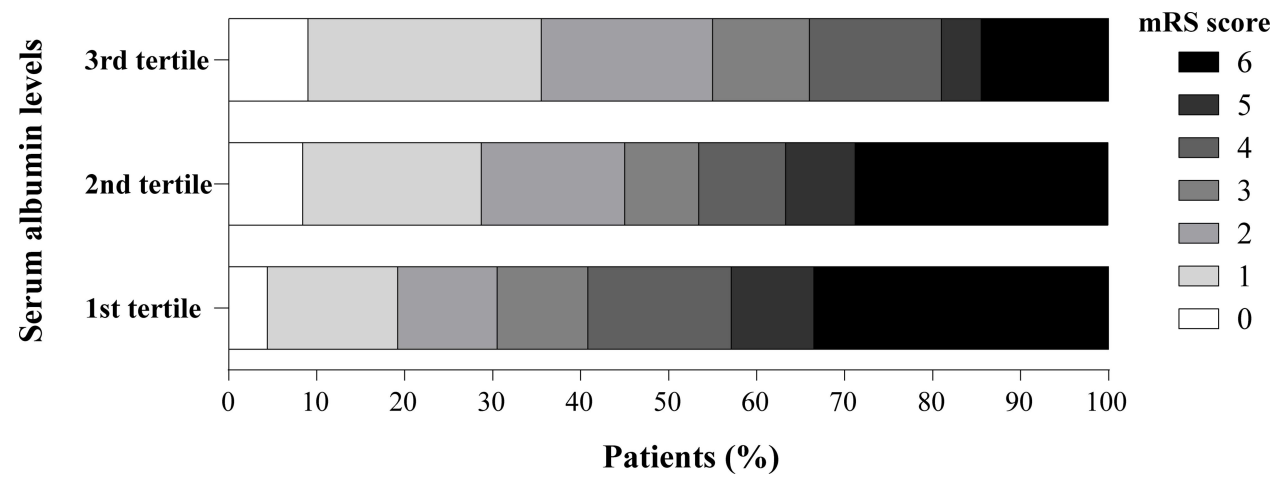

Figure I Distribution of modified Rankin scale (mRS) score at 90 days according to the albumin tertile. Adjusted odds ratio of ordinal logistic regression analysis for lowest vs highest tertile of serum albumin: $1.62(95 \% \mathrm{Cl}, 1.08-2.43 ; P=0.039$ for trend) for increased of mRS score. 
Table 2 Comparison of Baseline Characteristics According to Patients with and without Poor Functional Outcome

\begin{tabular}{|c|c|c|c|}
\hline \multirow[t]{2}{*}{ Variables } & \multicolumn{2}{|c|}{ Poor Functional Outcome } & \multirow[t]{2}{*}{$P$-value } \\
\hline & Yes, $n=342$ & No, $n=263$ & \\
\hline \multicolumn{4}{|l|}{ Demographic characteristics } \\
\hline Age, years & $67.4 \pm 11.4$ & $60.0 \pm 12.5$ & 0.001 \\
\hline Male, n (\%) & $180(52.6)$ & $179(68.1)$ & 0.001 \\
\hline \multicolumn{4}{|l|}{ Vascular risk factors, n (\%) } \\
\hline Hypertension & $219(64.0)$ & $155(58.9)$ & 0.201 \\
\hline Diabetes mellitus & $63(18.4)$ & $4 I(15.6)$ & 0.360 \\
\hline Hyperlipidemia & $26(7.6)$ & $28(10.6)$ & 0.193 \\
\hline Atrial fibrillation & $160(46.8)$ & $81(30.9)$ & 0.001 \\
\hline Coronary heart disease & $78(22.9)$ & $40(19.8)$ & 0.358 \\
\hline \multicolumn{4}{|l|}{ Clinical data } \\
\hline Systolic blood pressure, $\mathrm{mmHg}$ & $150.0 \pm 25.8$ & $142.1 \pm 25.5$ & 0.001 \\
\hline Diastolic blood pressure, $\mathrm{mmHg}$ & $85.0 \pm \mid 4.5$ & $83.3 \pm 13.6$ & 0.132 \\
\hline Time from onset to treatment, $\mathrm{min}$ & $270.0(207.0,352.0)$ & $258.0(190.0,340.0)$ & 0.064 \\
\hline Time from puncture to recanalization, $\mathrm{min}$ & $75.0(53.0,103.0)$ & $75.0(55.0,102.0)$ & 0.783 \\
\hline Baseline NIHSS, score & $18.0(14.0,23.0)$ & $13.0(10.0,18.0)$ & 0.001 \\
\hline Baseline ASPECTS, score & $9.0(7.0,10.0)$ & $10.0(9.0,10.0)$ & 0.001 \\
\hline Cause of stroke, n (\%) & & & 0.002 \\
\hline Atherosclerotic & $135(39.5)$ & $136(5 \mid .7)$ & \\
\hline Cardioembolic & $185(54.1)$ & $104(39.5)$ & \\
\hline Others & $22(6.4)$ & $23(8.7)$ & \\
\hline Poor collateral status & $136(40.0)$ & $182(69.7)$ & 0.001 \\
\hline Procedural modes, $\mathrm{n}(\%)$ & & & 0.488 \\
\hline Stent retriever only & $162(47.4)$ & $129(49.0)$ & \\
\hline Stent retriever with rescue therapy ${ }^{\mathrm{a}}$ & $180(52.6)$ & $134(51.0)$ & \\
\hline Passes of stent retriever & $1.0(1.0,2.0)$ & $\mathrm{I} .0(1.0,2.0)$ & 0.001 \\
\hline Vascular occlusion site, n (\%) & & & 0.007 \\
\hline Internal carotid artery & $157(45.9)$ & $92(35.0)$ & \\
\hline Middle cerebral artery & $185(54.1)$ & $171(65.0)$ & \\
\hline Prior IVT, n (\%) & $110(32.2)$ & $89(33.8)$ & 0.663 \\
\hline sICH, n (\%) & $84(24.6)$ & $9(3.4)$ & 0.001 \\
\hline Recanalization, n (\%) & $259(75.7)$ & 241 (9l.6) & 0.001 \\
\hline
\end{tabular}


Table 2 (Continued).

\begin{tabular}{|l|l|l|l|}
\hline Variables & Poor Functional Outcome & P-value \\
\cline { 2 - 4 } & Yes, $\mathbf{n = 3 4 2}$ & No, $\mathbf{n = 2 6 3}$ \\
\hline Laboratory data & & & \\
\hline Total cholesterol, $\mathrm{mmol} / \mathrm{L}$ & $4.3 \pm 1.1$ & $4.3 \pm 1.1$ & 0.878 \\
\hline Triglyceride, mmol/L & $1.0(0.7,1.5)$ & $1.1(0.8,1.5)$ & 0.581 \\
\hline Low-density lipoprotein, mmol/L & $2.4(1.9,3.1)$ & $2.5(2.0,3.1)$ & 0.382 \\
\hline High-density lipoprotein, $\mathrm{mmol} / \mathrm{L}$ & $1.2 \pm 0.4$ & $1.2 \pm 0.5$ & 0.893 \\
\hline Blood glucose, mmol/L & $8.3 \pm 3.9$ & $6.9 \pm 2.5$ & 0.001 \\
\hline Hs-CRP, mg/L & $10.1(2.9,29.0)$ & $5.8(2.1,13.9)$ & 0.006 \\
\hline Albumin, g/L & $38.0(35.0,40.7)$ & $39.5(37.4,41.6)$ & 0.001 \\
\hline
\end{tabular}

Note: ${ }^{a}$ Rescue therapy includes balloon angioplasty, permanent implantation of stent, intra-arterial thrombolysis or intra-arterial tirofiban infusion.

Abbreviations: ASPECTS, the Alberta Stroke Program Early Computed Tomography Score; Hs-CRP, hypersensitive C-reactive protein; IVT, intravenous thrombolysis; NIHSS, National Institutes of Health Stroke Scale; sICH, symptomatic intracranial hemorrhage.

Table 3 Odds Ratio and $95 \% \mathrm{Cl}$ of Albumin Levels with Clinical Outcomes

\begin{tabular}{|c|c|c|c|c|c|c|}
\hline & $\begin{array}{l}\text { Ist Tertile, } \\
<37.3 \mathrm{~g} / \mathrm{L}\end{array}$ & $\begin{array}{l}\text { 2nd Tertile, 37.3g/L } \\
-40.2 \mathrm{~g} / \mathrm{L}\end{array}$ & $\begin{array}{l}\text { 3rd Tertile, } \\
>40.2 \mathrm{~g} / \mathrm{L}\end{array}$ & $\begin{array}{l}P \text { for } \\
\text { Trend }\end{array}$ & $\begin{array}{l}\text { Albumin Levels } \\
\text { (Continuous) }\end{array}$ & $P$-value \\
\hline \multicolumn{7}{|c|}{ Poor functional outcome (mRS score of 3-6) } \\
\hline No. of cases (\%) & $14 \mid(69.5)$ & III (54.9) & $90(45.0)$ & & & \\
\hline Crude model & $2.78(1.85-4.18)$ & $1.49(1.01-2.21)$ & Ref & 0.001 & $0.91(0.87-0.94)$ & 0.001 \\
\hline Model I & $2.33(1.52-3.56)$ & $1.37(0.90-2.06)$ & Ref & 0.002 & $0.92(0.88-0.95)$ & 0.004 \\
\hline Model $2^{\mathrm{a}}$ & $2.43(1.18-5.01)$ & $1.28(0.65-2.54)$ & Ref & 0.046 & $0.89(0.84-0.96)$ & 0.011 \\
\hline \multicolumn{7}{|c|}{ Death (mRS score of 6) } \\
\hline No. of cases (\%) & $68(33.5)$ & $58(28.7)$ & $29(14.5)$ & & & \\
\hline Crude model & $2.97(1.82-4.85)$ & $2.38(1.44-3.91)$ & Ref & 0.001 & $0.92(0.89-0.96)$ & 0.001 \\
\hline Model I & $2.34(1.40-3.89)$ & $2.11(1.26-3.55)$ & Ref & 0.005 & $0.94(0.90-0.98)$ & 0.003 \\
\hline Model $2^{b}$ & $3.19(1.24-8.24)$ & $2.01(0.72-5.58)$ & Ref & 0.022 & $0.88(0.82-0.97)$ & 0.006 \\
\hline \multicolumn{7}{|c|}{ Symptomatic intracranial hemorrhage } \\
\hline No. of cases (\%) & $35(17.2)$ & $32(15.8)$ & $26(13.0)$ & & & \\
\hline Crude model & $1.39(0.8 \mathrm{I}-2.42)$ & $1.26(0.72-2.03)$ & Ref & 0.487 & $0.99(0.95-1.02)$ & 0.982 \\
\hline Model I & $1.31(0.75-2.29)$ & $1.22(0.70-2.15)$ & Ref & 0.633 & $1.00(0.96-1.06)$ & 0.801 \\
\hline Model $2^{c \S}$ & $1.30(0.66-2.18)$ & $1.19(0.65-2.17)$ & Ref & 0.797 & $1.02(0.96-1.07)$ & 0.571 \\
\hline
\end{tabular}

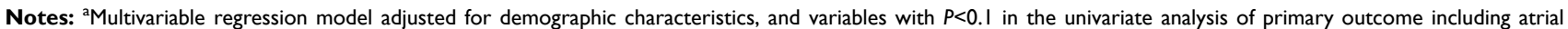
fibrillation, systolic blood pressure, baseline NIHSS score, baseline ASPECT score, onset to treatment time, stroke etiology, collateral status, passes of stent retriever, vascular occlusion site, prior IVT, sICH, recanalization, blood glucose, and Hs-CRP levels. 'Multivariable regression model adjusted for demographic characteristics, and variables with $P<0.1$ in the univariate analysis of mortality including atrial fibrillation, systolic blood pressure, baseline NIHSS score, baseline ASPECT score, stroke etiology, collateral status, passes of stent retriever, vascular occlusion site, prior IVT, sICH, recanalization, low-density lipoprotein, blood glucose, and Hs-CRP levels.

' ultivariable regression model adjusted for demographic characteristics, and variables with $\mathrm{P}<0 . \mathrm{I}$ in the univariate analysis of sICH including baseline $\mathrm{NIHSS}$ score, baseline ASPECT score, onset to treatment time, stroke etiology, collateral status, passes of stent retriever, recanalization, and blood glucose.

Abbreviations: ASPECTS, the Alberta Stroke Program Early Computed Tomography Score; Hs-CRP, hypersensitive C-reactive protein; IVT, intravenous thrombolysis; NIHSS, National Institutes of Health Stroke Scale; sICH, symptomatic intracranial hemorrhage. 

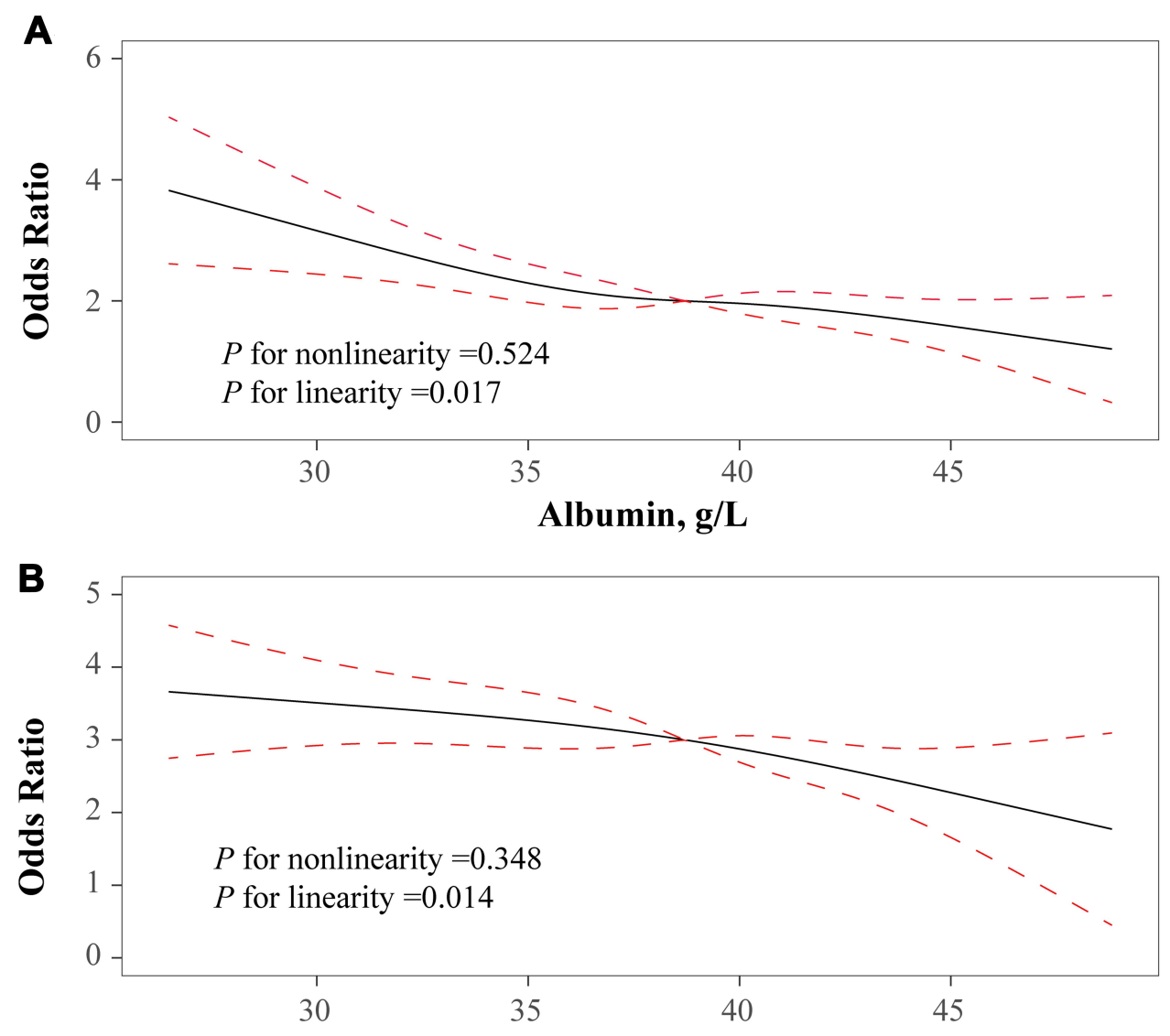

Albumin, g/L

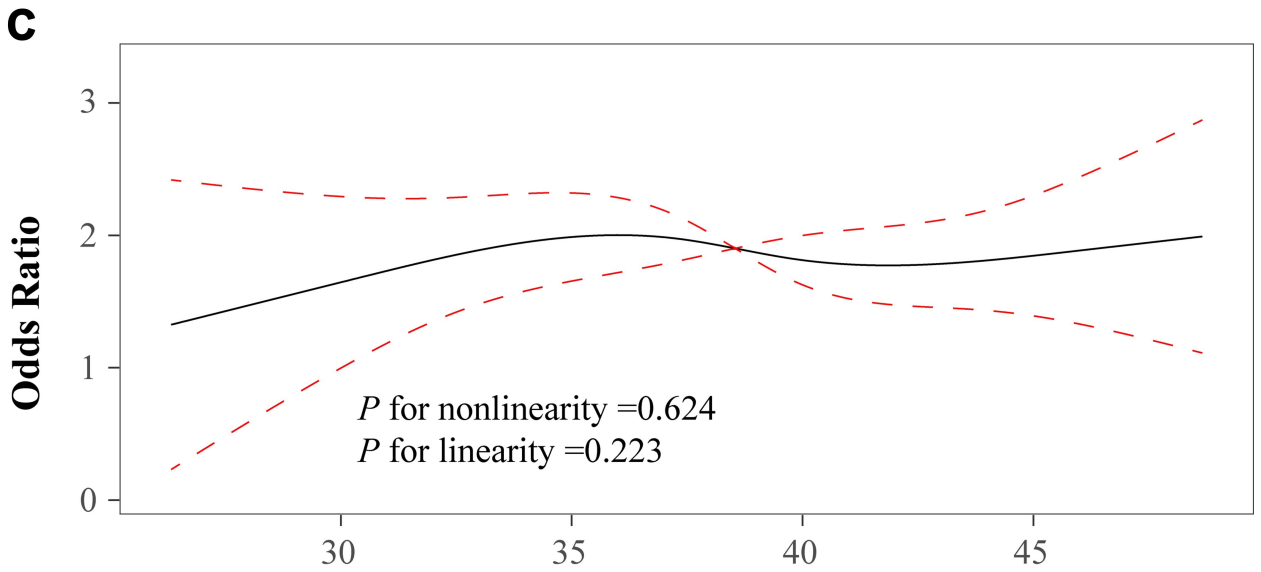

Albumin, g/L

Figure 2 Association of albumin levels with risk of poor functional outcome (A), death (B), and symptomatic intracranial hemorrhage (C). Odds ratio and $95 \% \mathrm{Cl}$ were derived from restricted cubic spline regression, with knots placed at 5th, 35th, 65th, and 95th percentiles of albumin levels. The reference point for albumin levels were the midpoint of the reference group from categorical analysis. Odds ratio were adjusted for the same variables as model 2 in Table 3.

insufficiency, hepatic disease, and heart failure. Decreased serum albumin levels have been reported to be associated with the severity of ischemic stroke for follow-up at 90 days. ${ }^{28}$ Furthermore, patients with hypoalbuminemia had a higher risk of mortality at six months after the first onset of stroke. ${ }^{14}$ As preclinical literatures showed convincing proof for the neuroprotection of albumin, several clinical trials have been performed to investigate the role of albumin in humans. However, Ginsberg et $\mathrm{al}^{17}$ did not find that $25 \%$ albumin can improve neurologic function in ischemic 


\begin{tabular}{|c|c|c|c|c|}
\hline \multirow[b]{2}{*}{ Subgroup } & \multirow[b]{2}{*}{ Cases } & \multicolumn{3}{|c|}{ Poor functional outcome } \\
\hline & & OR (95\%CI) & & $P$ for interaction \\
\hline \multicolumn{4}{|l|}{ Age } & 0.725 \\
\hline$>65$ years & 296 & $0.90(0.81-0.99)$ & $\longmapsto$ & \\
\hline$\leq 65$ years & 309 & $0.87(0.79-0.96)$ & $\longmapsto$ & \\
\hline \multicolumn{4}{|l|}{ Gender } & 0.077 \\
\hline Male & 359 & $0.90(0.82-0.99)$ & $\longmapsto$ & \\
\hline Female & 246 & $0.94(0.84-1.04)$ & $\longmapsto$ & \\
\hline \multicolumn{4}{|l|}{ Hypertension } & 0.521 \\
\hline Yes & 374 & $0.89(0.81-0.97)$ & $\longmapsto$ & \\
\hline No & 231 & $0.88(0.77-0.99)$ & $\longmapsto$ & \\
\hline \multicolumn{4}{|l|}{ Diabetes } & 0.699 \\
\hline Yes & 104 & $0.80(0.59-1.09)$ & - & \\
\hline No & 501 & $0.89(0.83-0.97)$ & $\mapsto$ & \\
\hline \multicolumn{4}{|l|}{ Atrial fibrillation } & 0.651 \\
\hline Yes & 241 & $0.83(0.73-0.96)$ & $\longmapsto$ & \\
\hline No & 364 & $0.90(0.82-0.99)$ & $\longmapsto$ & \\
\hline \multicolumn{4}{|l|}{ Prior IVT } & 0.422 \\
\hline Yes & 199 & $0.97(0.85-1.11)$ & $\longmapsto$ & \\
\hline No & 406 & $0.81(0.72-0.90)$ & $\longmapsto$ & \\
\hline \multicolumn{4}{|l|}{ Recanalization } & 0.96 \\
\hline Yes & 500 & $0.89(0.82-0.96)$ & $\longmapsto-$ & \\
\hline No & 105 & $0.92(0.68-1.25)$ & $\longrightarrow$ & \\
\hline \multicolumn{4}{|l|}{ OTT, min } & 0.813 \\
\hline$>270$ & 289 & $0.89(0.80-1.01)$ & $\longmapsto$ & \\
\hline$\leq 270$ & 316 & $0.88(0.81-0.97)$ & $\longmapsto$ & \\
\hline \multicolumn{4}{|l|}{ NIHSS, score } & 0.256 \\
\hline$>16$ & 289 & $0.88(0.82-0.94)$ & $\mapsto-1$ & \\
\hline$\leq 16$ & 316 & $0.96(0.90-1.03)$ & $\mapsto-1$ & \\
\hline \multicolumn{4}{|l|}{ ASPECT, score } & 0.644 \\
\hline$>8$ & 389 & $0.92(0.88-0.98)$ & $\mapsto$ & \\
\hline$\leq 8$ & 216 & $0.93(0.86-0.99)$ & $\longmapsto-1$ & \\
\hline \multicolumn{4}{|l|}{ Cause of stroke } & 0.374 \\
\hline Atherosclerotic & 271 & $0.92(0.86-0.98)$ & $\mapsto-1$ & \\
\hline \multirow[t]{2}{*}{ Cardioembolic } & 289 & $0.90(0.83-0.97)$ & $\mapsto$ & \\
\hline & & & 0.5 & 1.5 \\
\hline
\end{tabular}

Figure 3 Subgroup analyses of the association between albumin levels and primary outcome. ORs were calculated for increase in albumin levels after adjustment for the same variables as model 2 in Table 3, except for the stratified variable.

Abbreviations: ASPECTS, the Alberta Stroke Program Early Computed Tomography Score; IVT, intravenous thrombolysis; NIHSS, National Institutes of Health Stroke Scale; OTT, onset to treatment; sICH, symptomatic intracranial hemorrhage.

stroke patients. Further studies are needed focusing on the different groups of stroke patients. To the best of our knowledge, few researchers have investigated the correlation of albumin levels with prognosis in ischemic stroke patients after EVT. Our study extended the current knowledge about the neuroprotection of albumin in ischemic stroke as it demonstrated a negative association between albumin levels and poor prognosis in patients treated with EVT. Randomized controlled trials are recommended to detect the benefit of supplementation of albumin in ischemic stroke patients after EVT.

The mechanisms by which serum albumin affects clinical outcomes in ischemic stroke patients after EVT are not completely understood, but several underlying pathophysiological pathways have been postulated. The rate of infarct progression after large vessel occlusion stroke is highly variable and depends not only on the duration of arterial occlusion but also the ability to maintain collateral blood flow above the threshold of infarction. ${ }^{29}$ Moreover, several studies showed that hypotension may contribute to worse outcomes in ischemic stroke after endovascular therapy. ${ }^{30,31}$ As the important regulator of colloidal osmotic pressure, low serum albumin levels may reduce the collateral flow and induce a poor clinical outcome in large vessel occlusive stroke after EVT. Experimental studies found that albumin can not only decrease hematocrit level but also mediate erythrocyte aggregation by increasing low shear viscosity and reducing erythrocyte sedimentation under no-flow conditions, ${ }^{32}$ contributing to increase the cerebral blood flow. Sufficient blood perfusion for 
ischemic penumbra area might rescue more neuron and prevent infarct growth. Further studies with imaging data such as infarct progression and larger final infarct volumes are needed to clarify this potential mechanism. Other possible pathways include suppressing inflammatory response and oxidative stress, $^{12}$ inhibiting platelet aggregation, ${ }^{8,33}$ reducing the various cytokines adhesion within postcapillary microcirculation, ${ }^{34}$ and transporting the free fatty acids post-ischemia. ${ }^{35}$ In addition, ischemiainduced blood-brain barrier dysfunction facilitate albumin to penetrate into the brain parenchyma, where it may be taken up by cortical neurons with normal morphologic features. ${ }^{36}$ All these studies suggested that serum albumin could be beneficial in ischemic injury.

A major strength of the present study is that it included a relatively large number of EVT patients from 21 stroke centers across China. However, several limitations should be noted. First, this study was based on a registry that retrospectively collected patient related-data, which inevitably produced systematic bias. Second, serum albumin concentrations were evaluated only once after admission, so we were unable to explore the association between albumin changes and prognosis. Third, although we controlled for a large number of confounders, some potential confounders were not available, such as dietary intake and nutritional status. Finally, we did not compare albumin levels in serum with those in the cerebrospinal fluid. Therefore, the interpretation of our findings must be taken cautiously.

In conclusion, our present study showed that decreased albumin levels at baseline may increase the risk of poor prognosis after EVT in patients with acute anterior circulation large vessel occlusion stroke. Additional studies should be conducted to confirm whether these results are applicable to a much broader population.

\section{Data Availability}

The data that support the findings of this study are available on request from the corresponding author (Huaiming Wang).

\section{Funding}

This study was supported in part by the National Natural Science Foundation of China (No. 81870946; No. 81530038; No. 81771424).

\section{Disclosure}

The authors report no conflicts of interest in this work.

\section{References}

1. Jovin T, Chamorro A, Cobo E, et al. Thrombectomy within 8 hours after symptom onset in ischemic stroke. $N$ Engl $J$ Med. 2015;372 (24):2296-2306. doi:10.1056/NEJMoa1503780

2. Campbell B, Mitchell P, Kleinig T, et al. Endovascular therapy for ischemic stroke with perfusion-imaging selection. $N$ Engl J Med. 2015;372(11):1009-1018.

3. Goyal M, Demchuk A, Menon B, et al. Randomized assessment of rapid endovascular treatment of ischemic stroke. $N$ Engl $J$ Med. 2015;372(11):1019-1030.

4. Berkhemer O, Fransen P, Beumer D, et al. A randomized trial of intraarterial treatment for acute ischemic stroke. $N$ Engl $J$ Med. 2015;372(1):11-20

5. Saver J, Goyal M, Bonafe A, et al. Stent-retriever thrombectomy after intravenous t-pa vs. T-Pa Alone in Stroke. $N$ Engl J Med. 2015;372 (24):2285-2295.

6. Powers W, Rabinstein A, Ackerson T, et al. 2018 guidelines for the early management of patients with acute ischemic stroke: a guideline for healthcare professionals from the american heart association/ american stroke association. Stroke. 2018;49(3):e46-e110.

7. Goyal M, Menon B, van Zwam WH, et al. Endovascular thrombectomy after large-vessel ischaemic stroke: a meta-analysis of individual patient data from five randomised trials. Lancet. 2016;387(10029):1723-1731.

8. Maalej N, Albrecht R, Loscalzo J, et al. The potent platelet inhibitory effects of S-nitrosated albumin coating of artificial surfaces. $J \mathrm{Am}$ Coll Cardiol. 1999;33(5):1408-1414.

9. Danesh J, Collins R, Appleby P, et al. Association of fibrinogen, C-reactive protein, albumin, or leukocyte count with coronary heart disease: meta analyses of prospective studies. JAMA. 1998;279 (18):477-1482.

10. Roche M, Rondeau P, Singh N, et al. The antioxidant properties of serum albumin. FEBS Lett. 2008;582(13):1783-1787. doi:10.1016/j. febslet.2008.04.057

11. O E. Decreased serum albumin predicts hypertension in a Japanese health screening population. Internal Med. 2014;53(7):655-660. doi:10.2169/internalmedicine.53.1894

12. Ishizaka $\mathrm{N}$, Ishizaka $\mathrm{Y}$, Nagai $\mathrm{R}$, Toda $\mathrm{E}$, Hashimoto $\mathrm{H}$, Yamakado M. Association between serum albumin, carotid atherosclerosis, and metabolic syndrome in Japanese individuals. Atherosclerosis. 2007;193:373-379.

13. Arques S. Human serum albumin in cardiovascular diseases. Eur J Intern Med. 2018;52:8-12.

14. Chakraborty B, Vishnoi G, Goswami B, et al. Lipoprotein(a), ferritin, and albumin in acute phase reaction predicts severity and mortality of acute ischemic stroke in North Indian Patients. J Stroke Cerebrovasc Dis. 2013;22(7):e159-67.

15. Babu M, Kaul S, Dadheech S, et al. Serum albumin levels in ischemic stroke and its subtypes: correlation with clinical outcome. Nutrition. 2013;29(6):872-875.

16. Sutherland B, Minnerup J, Balami J, et al. Neuroprotection for ischaemic stroke: translation from the bench to the bedside. Int J Stroke. 2012;7(5):407-418.

17. Ginsberg M, Palesch Y, Hill M, et al. High-dose albumin treatment for acute ischaemic stroke (ALIAS) Part 2: a randomised, double-blind, Phase 3, placebo-controlled trial. Lancet Neurol. 2013;12(11):1049-1058.

18. Zi W, Wang H, Yang D, et al. Clinical effectiveness and safety outcomes of endovascular treatment for acute anterior circulation ischemic stroke in China. Cerebrovasc Dis. 2017;44(5-6):248-258.

19. Brott T, Adams HP Jr, Olinger CP, et al. Measurements of acute cerebral infarction: a clinical examination scale. Stroke. 1989;20(7):864-870.

20. Adams HJ, Bendixen B, Kappelle L, et al. Classification of subtype of acute ischemic stroke definitions for use in a multicenter clinical trial TOAST Trial of Org 10172 in acute stroke treatment. Stroke. 1993;24(1):35-41. 
21. Zaidat O, Yoo A, Khatri P, et al. Recommendations on angiographic revascularization grading standards for acute ischemic stroke: a consensus statement. Stroke. 2013;44(9):2650-2663.

22. Zhang X, Yuan K, Wang H, et al. Nomogram to predict mortality of endovascular thrombectomy for ischemic stroke despite successful recanalization. J Am Heart Assoc. 2020;9(3):e014899.

23. von Kummer R, Broderick JP, Campbell BC, et al. The Heidelberg bleeding classification: classification of bleeding events after ischemic stroke and reperfusion therapy. Stroke. 2015;46(10):29 81-2986.

24. Durrleman S, Simon R. Flexible regression models with cubic splines. Stat Med. 1989;8(5):551-561.

25. Davalos A, Ricart W, Gonzalez-Huix F, et al. Effect of malnutrition after acute stroke on clinical outcome. Stroke. 1996;27(6):10 28-1032.

26. Dziedzic T, Pera J, Slowik A, et al. Hypoalbuminemia in acute ischemic stroke patients: frequency and correlates. Eur J Clin Nutr. 2007;61:1318-1322.

27. Davis J, Wong A, Schluter P, et al. Impact of premorbid undernutrition on outcome in stroke patients. Stroke. 2004;35:1930-1934.

28. Dziedzic T, Slowik A, Szczudlik A. Serum albumin level as a predictor of ischemic stroke outcome. Stroke. 2004;35(8):156-158.

29. Rocha M, Jovin T. Fast versus slow progressors of infarct growth in large vessel occlusion stroke: clinical and research implications. Stroke. 2017;48(9):2621-2627.
30. Löwhagen Hendén P, Rentzos A, Karlsson JE, et al. Hypotension during endovascular treatment of ischemic stroke is a risk factor for poor neurological outcome. Stroke. 2015;46(9):2678-2680.

31. Jagani M, Brinjikji W, Rabinstein A, et al. Hemodynamics during anesthesia for intra-arterial therapy of acute ischemic stroke. J Neurointerv Surg. 2016;8(9):883-888.

32. Reinhart W, Nagy C. Albumin affects erythrocyte aggregation and sedimentation. Eur J Clin Invest. 1995;25(7):523-528.

33. George G, Alastair GS. Albumin inhibits platelet-activating factor (PAF)-induced responses in platelets and macrophages: implications for the biologically active form of PAF. Br J Pharmacol. 1992;107 (1):73-77.

34. Belayev L, Pinard E, Nallet H, et al. Albumin therapy of transient focal cerebral ischemia: in vivo analysis of dynamic microvascular responses. Stroke. 2002;33(4):1077-1084

35. Rodriguez de Turco EB, Belayev L, Liu Y, et al. Systemic fatty acid responses to transient focal cerebral ischemia: influence of neuroprotectant therapy with human albumin. $J$ Neurochem. 2002;83 (3):515-524

36. Remmers M, Schmidt-Kastner R, Belayev L, et al. Protein extravasation and cellular uptake after high-dose human-albumin treatment of transient focal cerebral ischemia in rats. Brain Res. 1999;827 (1-2):237-242.
Neuropsychiatric Disease and Treatment

\section{Publish your work in this journal}

Neuropsychiatric Disease and Treatment is an international, peerreviewed journal of clinical therapeutics and pharmacology focusing on concise rapid reporting of clinical or pre-clinical studies on a range of neuropsychiatric and neurological disorders. This journal is indexed on PubMed Central, the 'PsycINFO' database and CAS, and
Dovepress

is the official journal of The International Neuropsychiatric Association (INA). The manuscript management system is completely online and includes a very quick and fair peer-review system, which is all easy to use. Visit http://www.dovepress.com/testimonials.php to read real quotes from published authors. 